LBL-37697

UCB-PTH-95/31

ITP-SB-95-38

hep-th/9606135

June 19, 2018

\title{
SUPERGRAVITY COUPLED TO CHIRAL AND YANG-MILLS MATTER AT ONE LOOPA
}

\author{
Mary K. Gaillard ${ }^{a}$, Vidyut Jain ${ }^{b}$ and Kamran Saririan ${ }^{a}$ \\ ${ }^{a}$ Physics Department and Theoretical Physics Group, Lawrence Berkeley \\ Laboratory, University of California, Berkeley, California 94720 \\ ${ }^{b}$ Institute for Theoretical Physics, SUNY at Stony Brook, NY 11794
}

\begin{abstract}
We present the full result for the divergent one-loop contribution to the effective boson Lagrangian for supergravity coupled to chiral and Yang-Mills supermultiplets. We also consider the specific case of dilaton couplings in effective supergravity Lagrangians from superstrings, for which the one-loop result is considerably simplified.
\end{abstract}

*This work was supported in part by the Director, Office of Energy Research, Office of High Energy and Nuclear Physics, Division of High Energy Physics of the U.S. Department of Energy under Contract DE-AC03-76SF00098 and in part by the National Science Foundation under grants PHY-95-14797 and PHY-93-09888.

†Present address: vid@dotcomdev.com 


\section{Disclaimer}

This document was prepared as an account of work sponsored by the United States Government. While this document is believed to contain correct information, neither the United States Government nor any agency thereof, nor The Regents of the University of California, nor any of their employees, makes any warranty, express or implied, or assumes any legal liability or responsibility for the accuracy, completeness, or usefulness of any information, apparatus, product, or process disclosed, or represents that its use would not infringe privately owned rights. Reference herein to any specific commercial products process, or service by its trade name, trademark, manufacturer, or otherwise, does not necessarily constitute or imply its endorsement, recommendation, or favoring by the United States Government or any agency thereof, or The Regents of the University of California. The views and opinions of authors expressed herein do not necessarily state or reflect those of the United States Government or any agency thereof, or The Regents of the University of California.

Lawrence Berkeley Laboratory is an equal opportunity employer. 
In this Letter we present the ultraviolet divergent contributions [1, 2] to the one-loop corrections for a general supergravity theory [3, 4], with only the restriction that the gauge kinetic function is diagonal in gauge indices: $f_{a b}(Z)=\delta_{a b} k_{a} f(Z)$. The details of the calculations are given in [1, 2]. Here we give only sufficient details to establish notation and to specify the gauge fixing and our prescriptions for the loop expansion of the effective action.

The tree-level supergravity Lagrangian [3, 4] we adopt, with $f(z)=x+i y$ (which is trivially generalized [2] to $f_{a b}=\delta_{a b} k_{a} f, k_{a}=$ constant, and so includes all known string models), is

$$
\begin{aligned}
\frac{1}{\sqrt{g}} \mathcal{L}= & \frac{1}{2} r-\frac{x}{4} F_{\mu \nu} F^{\mu \nu}-\frac{y}{4} \tilde{F}_{\mu \nu} F^{\mu \nu}+K_{i \bar{m}} \mathcal{D}^{\mu} z^{i} \mathcal{D}_{\mu} \bar{z}^{\bar{m}}-V \\
& +\frac{i x}{2} \bar{\lambda} \not D \lambda+i K^{i \bar{m}}\left(\bar{\chi}_{L}^{\bar{m}} \not D \chi_{L}^{i}+\bar{\chi}_{R}^{i} \not D \chi_{R}^{\bar{m}}\right) \\
& +e^{-K / 2}\left(\frac{1}{4} f_{i} \bar{A}^{i} \bar{\lambda}_{R} \lambda_{L}-A_{i j} \bar{\chi}_{R}^{i} \chi_{L}^{j}+\text { h.c. }\right) \\
& +\frac{1}{4} \bar{\psi}_{\mu} \gamma^{\nu}(i \not D+M) \gamma^{\mu} \psi_{\nu}-\frac{1}{4} \bar{\psi}_{\mu} \gamma^{\mu}(i \not D+M) \gamma^{\nu} \psi_{\nu} \\
& -\left[\frac{x}{8} \bar{\psi}_{\mu} \sigma^{\nu \rho} \gamma^{\mu} \lambda_{a} F_{\nu \rho}^{a}+\bar{\psi}_{\mu} \not \bar{z}^{\bar{m}} K_{i \bar{m}} \gamma^{\mu} L \chi^{i} \frac{1}{4} \bar{\psi}_{\mu} \gamma^{\mu} \gamma_{5} \lambda^{a} \mathcal{D}_{a}+i \bar{\psi}_{\mu} \gamma^{\mu} L \chi^{i} m_{i}+\text { h.c. }\right] \\
& +\left(i \bar{\lambda}_{R}^{a}\left[2 K_{i \bar{m}}\left(T_{a} \bar{z}\right)^{\bar{m}}-\frac{1}{2 x} f_{i} \mathcal{D}_{a}-\frac{1}{4} \sigma_{\mu \nu} F_{a}^{\mu \nu} f_{i}\right] \chi_{L}^{i}+\text { h.c. }\right) \\
& +4 \text { fermion terms, }
\end{aligned}
$$

where

$$
\begin{aligned}
V & =\hat{V}+\mathcal{D}, \quad \hat{V}=e^{-K}\left(A_{i} \bar{A}^{i}-3 A \bar{A}\right), \quad \mathcal{D}=\frac{1}{2 x} \mathcal{D}_{a} \mathcal{D}^{a}, \quad \mathcal{D}_{a}=K_{i}\left(T^{a} z^{i}\right), \\
\bar{M} & =(M)^{\dagger}=e^{K / 2}(W R+\bar{W} L) \\
A & =e^{K} W, \quad \bar{A}=e^{K} \bar{W}, \quad m_{i}=e^{-K / 2} A_{i} .
\end{aligned}
$$

$K(z, \bar{z})$ is the Kähler potential, $W(z)$ is the superpotential, $T^{a}$ is a generator of the gauge group, and

$$
A_{i_{1} \cdots i_{n}}=D_{i_{1}} \cdots D_{i_{n}} A, \quad \bar{A}^{i_{1} \cdots i_{n}}=D^{i_{1}} \cdots D^{i_{n}} \bar{A}, \quad D^{i}=K^{i \bar{m}} D_{\bar{m}}
$$


with $D_{i}$ the scalar field reparameterization covariant derivative, and $K^{i \bar{m}}$ the inverse Kähler metric. The one-loop effective action is determined from the quadratic quantum action:

$$
\begin{aligned}
\mathcal{L}_{\text {quad }} & (\Phi, \Theta, c)=\frac{1}{2} \hat{\phi}^{I} \hat{\phi}^{J}\left(\partial_{I} \partial_{J}+\left(A_{I}\right)_{J}^{K} \partial_{K}\right) S+\mathcal{L}_{g f}+\mathcal{L}_{g h}= \\
& -\frac{1}{2} \Phi^{T} Z^{\Phi}\left(\hat{D}_{\Phi}^{2}+H_{\Phi}\right) \Phi+\frac{1}{2} \bar{\Theta} Z^{\Theta}\left(i \not D_{\Theta}-M_{\Theta}\right) \Theta \\
+ & \frac{1}{2} \bar{c} Z^{c}\left(\hat{D}_{c}^{2}+H_{c}\right) c+O\left(\psi_{c l}\right),
\end{aligned}
$$

where $\phi^{I}=\Phi^{I}, \Theta^{I}, \partial_{I}=\partial / \partial \phi^{I}$, and the column vectors,

$$
\Phi^{T}=\left(h_{\mu \nu}, \hat{\mathcal{A}}^{a}, \hat{z}^{i}, \hat{z}^{\bar{m}}\right), \quad \Theta^{T}=\left(\psi_{\mu}, \lambda^{a}, \chi_{L}^{i}, \chi_{R}^{\bar{m}}, \alpha\right), \quad c^{T}=\left(c_{\nu}, c^{a}, c_{\alpha}\right)
$$

represent the boson, fermion and ghost quantum degrees of freedom, respectively, with $\alpha=-C \bar{\alpha}^{T}$ an auxiliary field introduced [1] to implement gravitino gauge fixing. The connection $\left(A_{I}\right)_{J}^{K}$ in $(4)$, which is defined explicitly in [1, 2], is chosen so as to preserve all bosonic symmetries, and also to simplify matrix elements involving the graviton. In particular the quantum variables $\hat{z}^{i}, \hat{z}^{\bar{m}}$ are normal coordinates in the space of scalar fields: $\left(A_{i}\right)_{j}^{k}=\Gamma_{i j}^{k}$ is the affine connection associated with the Kähler metric $K_{i \bar{m}}$, giving a scalar field reparameterization invariant expansion. In (4) $\psi_{c l}$ represents background fermion fields that we set to zero; that is, we calculate only the one-loop bosonic action.

For the boson sector, we use a smeared gauge-fixing:

$$
\begin{aligned}
\mathcal{L} & \rightarrow \mathcal{L}+\mathcal{L}_{g f}, \quad \mathcal{L}_{g f}=-\frac{\sqrt{g}}{2} C_{A} Z^{A B} C_{B}, \\
Z & =\left(\begin{array}{cc}
\delta^{a b} & 0 \\
0 & -g^{\mu \nu}
\end{array}\right), \quad C=\left(\begin{array}{l}
C_{a} \\
C_{\mu}
\end{array}\right) .
\end{aligned}
$$

The Yang-Mills gauge-fixing term:

$$
C^{a}=\left(\mathcal{D}_{\mu}+\frac{\partial_{\mu} x}{2 x}\right) \hat{\mathcal{A}}_{\mu}^{a}+\frac{i}{\sqrt{x}} K_{i \bar{m}}\left[\left(T^{a} \bar{z}\right)^{\bar{m}} \hat{z}^{i}-\left(T^{a} z\right)^{i} \hat{z}^{\bar{m}}\right],
$$


preserves off-shell supersymmetry [5] in the limit of global supersymmetry and coincides with the string-loop result [6] for chiral multiplet wave function renormalization. The graviton gauge-fixing term:

$$
\sqrt{2} C_{\mu}=\left(\nabla^{\nu} h_{\mu \nu}-\frac{1}{2} \nabla_{\mu} h_{\nu}^{\nu}-2 \mathcal{D}_{\mu} z^{I} Z_{I J} \hat{z}^{J}+2 \mathcal{F}_{\mu \nu}^{a} \hat{\mathcal{A}}_{a}^{\nu}\right)
$$

is the one originally introduced by 't Hooft and Veltman [7], generalized [1] to include the Yang-Mills sector. The script quantum and classical Yang-Mills fields and field strengths are canonically normalized [8]:

$\mathcal{A}_{\mu}=\sqrt{x} A_{\mu}, \quad \hat{\mathcal{A}}_{\mu}=\sqrt{x} \hat{A}_{\mu}, \quad \mathcal{F}_{\mu \nu}=\sqrt{x} F_{\mu \nu}, \quad \sqrt{x} \mathcal{D}_{\mu} A_{\nu}=\left(\mathcal{D}_{\mu}-\frac{\partial_{\mu} x}{2 x}\right) \mathcal{A}_{\mu}$ where $\mathcal{D}_{\mu}$ is the gauge and general covariant derivative. In the earlier literature two gravitino gauge fixing procedures have been used: a) a Landau-type gauge [9, 10] $\gamma \cdot \psi=0$, implemented by the introduction of an auxiliary field, and b) a smeared gauge 11] $\mathcal{L} \rightarrow \mathcal{L}-\bar{F} \mathcal{M} F, F=\gamma \cdot \psi, \mathcal{M}=\frac{1}{4}(i \not D+2 M)$ supplemented with Nielsen-Kallosh ghosts. Here we use an unsmeared gauge $G=0$, with the gauge-fixing function [回]

$$
\begin{aligned}
G= & -\gamma^{\nu}(i \not D-\bar{M}) \psi_{\nu}-2\left(\not \supset z^{i} K_{i \bar{m}} R \chi^{\bar{m}}+\not \bar{D}^{\bar{m}} K_{i \bar{m}} L \chi^{i}\right) \\
& +\frac{x}{2} \sigma^{\nu \rho} \lambda_{a} F_{\nu \rho}^{a}+2 i m_{I} \chi^{I}-\gamma_{5} \mathcal{D}_{a} \lambda^{a}
\end{aligned}
$$

where $D_{\mu}$ contains the spin and chiral Kähler connections. The quantum Lagrangian is obtained by the introduction of an auxiliary field $\alpha: \delta(G)=$ $\int d \alpha \exp (i \alpha G)$, and a shift in the gravitino field: $\psi^{\prime}=\psi+\gamma \alpha, \bar{\psi}^{\prime}=\bar{\psi}+\bar{\alpha} \gamma$, so as to diagonalize the gravitino kinetic energy term. The ghost and ghostino determinants are obtained in the usual way as, respectively:

$$
\left(\hat{D}_{c}^{2}+H_{c}\right)_{B}^{A}=\frac{\partial}{\partial \epsilon_{A}} \delta C_{B}, \quad A, B=a, \mu, \quad\left(\hat{D}_{c}^{2}+H_{c}\right)_{\beta}^{\alpha}=\frac{\partial \delta G^{\alpha}}{\partial \epsilon^{\beta}},
$$

where $\hat{D}_{\mu}$ is related to $\mathcal{D}_{\mu}$ or $D_{\mu}$ by additional connections [1, 2]. With these choices the one-loop bosonic action takes a very simple form:

$$
S_{1}=\frac{i}{2} \operatorname{Tr} \ln \left(\hat{D}_{\Phi}^{2}+H_{\Phi}\right)-\frac{i}{2} \operatorname{Tr} \ln \left(-i \not D_{\Theta}+M_{\Theta}\right)+\frac{i}{2} \operatorname{STr} \ln \left(\hat{D}_{c}^{2}+H_{c}\right),
$$


where

$$
\mathrm{STr} \ln \left(\hat{D}_{c}^{2}+H_{c}\right)=2 \operatorname{Tr} \ln \left(\hat{D}_{c}^{2}+H_{c}\right)_{c_{\alpha}}-2 \operatorname{Tr} \ln \left(\hat{D}_{c}^{2}+H_{c}\right)_{c_{a, \mu}},
$$

which just reduces to determinants of the form of those for scalars and spin- $\frac{1}{2}$ fermions. Moreover the ghost and nonghost sectors have separately supersymmetric quantum spectra, except for the Yang-Mills fields:

$\frac{1}{2}(\operatorname{Tr} 1)_{\Theta}=(\operatorname{Tr} 1)_{\Phi}-2 N_{G}=2 N+2 N_{G}+10 . \quad(\operatorname{Tr} 1)_{c_{\alpha}}=4, \quad(\operatorname{Tr} 1)_{c_{a, b}}=4+N_{G}$,

where $N\left(N_{G}\right)$ is the number of chiral (gauge) supermultiplets. To evaluate (7) we separate [10, 2] the fermion determinant into helicity-even and -odd parts:

$$
-\frac{i}{2} \operatorname{Tr} \ln \left(-i \not D+M_{\Theta}\right) \equiv-\frac{i}{2} \operatorname{Tr} \ln \mathcal{M}\left(\gamma_{5}\right)=T_{-}+T_{+},
$$

where here $D_{\mu}$ contains all fermion connections, and

$$
\begin{aligned}
T_{-} & =-\frac{i}{4}\left[\operatorname{Tr} \ln \mathcal{M}\left(\gamma_{5}\right)-\operatorname{Tr} \ln \mathcal{M}\left(-\gamma_{5}\right)\right] \\
T_{+} & =-\frac{i}{4}\left[\operatorname{Tr} \ln \mathcal{M}\left(\gamma_{5}\right)+\operatorname{Tr} \ln \mathcal{M}\left(-\gamma_{5}\right)\right] \\
\mathcal{M}\left(\gamma_{5}\right) & =\gamma_{0}\left(-i \not D+M_{\Theta}\right)=\left(\begin{array}{cc}
\sigma_{+}^{\mu} D_{\mu}^{+} & M^{+} \\
M^{-} & \sigma_{-}^{\mu} D_{\mu}^{-}
\end{array}\right), \quad \sigma_{ \pm}^{\mu}=(1, \pm \vec{\sigma}) .
\end{aligned}
$$

Then defining

$$
\hat{D}_{\Theta}^{2}+H_{\Theta} \equiv\left(-i \not D_{\Theta}+M_{\Theta}\right)\left(i \not D_{\Theta}+M_{\Theta}\right)
$$

The one-loop bosonic action (7) reduces to:

$$
S_{1}=\frac{i}{2} \mathrm{~S} \operatorname{Tr} \ln \left(\hat{D}^{2}+H\right)+T_{-} .
$$

The helicity-odd term $T_{-}$is at most logarithmically divergent, and is finite [2] in the absence of a dilaton, that is, for $f(Z)=g^{-2}+i \theta / 8 \pi^{2}=$ constant. As discussed in 112, 2] there is an ambiguity in the separation (9) of the fermion 
determinant into helicity-even and -odd contributions, because terms that are even and odd in $\gamma_{5}$ can be interchanged by the use of the identities:

$$
\gamma_{5}=(i / 24) \epsilon^{\mu \nu \rho \sigma} \gamma_{\mu} \gamma_{\nu} \gamma_{\rho} \gamma_{\sigma}, \quad \sigma_{\mu \nu}=i \gamma_{5} \sigma^{\rho \sigma} \epsilon_{\rho \sigma \mu \nu}, \quad \text { etc. }
$$

In most cases the choice is dictated by gauge or Kähler covariance. However supersymmetry must be used to fix the off-diagonal gaugino- $\alpha$ and gauginodilaton mass terms:

$$
M_{\alpha \lambda^{a}}=-\sqrt{\frac{x}{2}} F_{a}^{\mu \nu} \sigma_{\mu \nu}, \quad M_{\chi^{i} \lambda^{a}}=-i \frac{f_{i}}{4 \sqrt{x}}\left(F_{a}^{\mu \nu}-i \gamma_{5} \tilde{F}_{a}^{\mu \nu}\right) \sigma_{\mu \nu}
$$

and the dilaton-dependent gaugino connection:

$$
A_{\lambda^{a} \lambda^{b}}^{\mu}=\delta_{a b} \frac{\partial^{\mu} y}{2 x} \frac{\epsilon^{\lambda \nu \rho \sigma}}{24} \gamma_{\lambda} \gamma_{\nu} \gamma_{\rho} \gamma_{\sigma}
$$

Eqs. (11) and (12) are precisely the choices that allow Pauli-Villars regularization of the quadratic divergences [12]. The choice (12) further insures the nonrenormalization [13] of the topological charge $\theta=8 \pi^{2} y$, and is consistent with linear-chiral multiplet duality [14] for the dilaton supermultiplet.

With the above conventions we obtain [1, 2] the following result for the divergent contributions to the one-loop corrected effective supergravity Lagrangian:

$$
\begin{aligned}
\mathcal{L}_{\text {eff }}= & \mathcal{L}(K)+\mathcal{L}_{1-\text { loop }}=\mathcal{L}\left(K_{R}\right)+\sqrt{g} \frac{\Lambda^{2}}{32 \pi^{2}} L_{0}+\sqrt{g} \frac{\ln \Lambda^{2}}{32 \pi^{2}}\left(L_{1}+L_{2}+N_{G} L_{g}\right), \\
L_{0}= & \frac{r}{2}\left(N_{G}-1\right)+2 K_{i \bar{m}}\left(\mathcal{D}_{\mu} z^{i} \mathcal{D}^{\mu} \bar{z}^{\bar{m}}+5 F^{i} \bar{F}^{\bar{m}}\right)-8 \mathcal{D}+\frac{28}{9} M \bar{M}+2 x^{-1} \mathcal{D}_{a} D_{i}\left(T^{a} z\right)^{i} \\
& +2 N\left(K_{i \bar{m}} F^{i} \bar{F}^{\bar{m}}-\frac{2}{9} M \bar{M}-\frac{r}{4}\right)-\left(N_{G} \frac{f_{i} \bar{f}_{\bar{m}}}{2 x^{2}}+2 R_{i \bar{m}}\right)\left(F^{i} \bar{F}^{\bar{m}}+\mathcal{D}_{\mu} z^{i} \mathcal{D}^{\mu} \bar{z}^{\bar{m}}\right), \\
L_{1}= & \frac{N+5}{27}\left[K_{i \bar{m}}\left(2 F^{i} \bar{F}^{\bar{m}}-\mathcal{D}_{\mu} z^{j} \mathcal{D}^{\mu} \bar{z}^{\bar{n}}\right) M \bar{M}-\frac{1}{3}(M \bar{M})^{2}\right] \\
& +\frac{N+5}{3} K_{i \bar{m}} K_{j \bar{n}}\left(\mathcal{D}_{\mu} z^{j} \mathcal{D}^{\mu} \bar{z}^{\bar{m}} F^{j} \bar{F}^{\bar{n}}+\mathcal{D}_{\mu} z^{j} \mathcal{D}^{\mu} z^{i} \mathcal{D}_{\nu} \bar{z}^{\bar{m}} \mathcal{D}^{\nu} \bar{z}^{\bar{n}}\right) \\
& +8\left(K_{j \bar{n}} F^{j} \bar{F}^{\bar{n}}-\frac{1}{3} M \bar{M}\right)\left[K_{i \bar{m}}\left(F^{i} \bar{F}^{\bar{m}}+\mathcal{D}_{\mu} z^{j} \mathcal{D}^{\mu} \bar{z}^{\bar{n}}\right)-\frac{1}{3} M \bar{M}\right]
\end{aligned}
$$




$$
\begin{aligned}
& -4\left(\mathcal{D}_{\mu} \bar{z}^{\bar{m}} \mathcal{D}^{\mu} z^{i} K_{i \bar{m}}\right)^{2}-8 \mathcal{D}_{\mu} z^{j} \mathcal{D}^{\mu} z^{i} \mathcal{D}_{\nu} \bar{z}^{\bar{m}} \mathcal{D}^{\nu} \bar{z}^{\bar{n}} K_{i \bar{n}} K_{j \bar{m}} \\
& -\left(\mathcal{D}_{\mu} z^{i} \mathcal{D}^{\mu} \bar{z}^{\bar{m}}+F^{i} \bar{F}^{\bar{m}}\right)\left[\frac{2}{3} K_{i \bar{m}} R_{j \bar{n}}\left(\mathcal{D}_{\mu} z^{j} \mathcal{D}^{\mu} \bar{z}^{\bar{n}}+F^{j} \bar{F}^{\bar{n}}\right)+4 R_{\bar{n} i \bar{m} j} \bar{F}^{\bar{n}} F^{j}\right] \\
& +\frac{1}{3} \mathcal{D}_{\mu} z^{i} \mathcal{D}_{\nu} \bar{z}^{\bar{m}} K_{i \bar{m}}\left(R_{j \bar{n}}-2 K_{j \bar{n}}\right)\left(\mathcal{D}^{\mu} z^{j} \mathcal{D}^{\nu} \bar{z}^{\bar{n}}-\mathcal{D}^{\nu} z^{j} \mathcal{D}^{\mu} \bar{z}^{\bar{n}}\right) \\
& -\left\{\left(\mathcal{D}_{\mu} z^{i} \mathcal{D}^{\mu} \bar{z}^{\bar{m}}+F^{i} \bar{F}^{\bar{m}}\right)\left[e^{-K} R_{n i}^{j k} A_{j k} \bar{A}_{\bar{m}}^{n}+e^{-K / 2}\left(D_{\bar{m}} R_{n i}^{j k}\right) A_{j k} F^{n}\right]+\text { h.c. }\right\} \\
& -e^{-K / 2}\left[\frac{1}{3} R_{j i}^{k \ell} A_{k \ell}\left(\bar{M} \mathcal{D}_{\mu} z^{i} \mathcal{D}^{\mu} z^{j}+M F^{i} F^{j}\right)\right. \\
& \left.+\mathcal{D}_{\mu} z^{i} \mathcal{D}^{\mu} z^{j} F^{m}\left(R_{j m}^{k \ell} A_{i k \ell}-R_{j i}^{k \ell} A_{m k \ell}\right)+\text { h.c. }\right] \\
& +R^{k}{ }_{j i}^{\ell} R_{\bar{n} k \bar{m} \ell}\left(\mathcal{D}_{\mu} z^{j} \mathcal{D}^{\mu} z^{i} \mathcal{D}_{\nu} \bar{z}^{\bar{n}} \mathcal{D}^{\nu} \bar{z}^{\bar{m}}-F^{j} F^{i} \bar{F}^{\bar{n}} \bar{F}^{\bar{m}}\right) \\
& +R_{j \bar{m} i}^{k} R_{\ell \bar{n} k}^{i}\left(\mathcal{D}_{\mu} z^{j} \mathcal{D}^{\mu} \bar{z}^{\bar{m}} \mathcal{D}_{\nu} z^{\ell} \mathcal{D}^{\nu} \bar{z}^{\bar{n}}+2 \mathcal{D}_{\mu} z^{j} \mathcal{D}^{\mu} \bar{z}^{\bar{m}} F^{\ell} \bar{F}^{\bar{n}}+F^{j} \bar{F}^{\bar{m}} F^{\ell} \bar{F}^{\bar{n}}\right) \\
& +\mathcal{D}_{\mu} z^{j} \mathcal{D}_{\nu} \bar{z}^{\bar{m}} R_{i \bar{m} j}^{k} \mathcal{D}^{\mu} z^{\ell} \mathcal{D}^{\nu} \bar{z}^{\bar{n}} R_{k \bar{n} \ell}^{i}-\mathcal{D}_{\mu} z^{j} \mathcal{D}_{\nu} \bar{z}^{\bar{m}} R_{i \bar{m} j}^{k} \mathcal{D}^{\nu} z^{\ell} \mathcal{D}^{\mu} \bar{z}^{\bar{n}} R_{k \bar{n} \ell}^{i}, \\
& L_{2}=\left[\mathcal{W}^{a b}\left(3 C_{G} \delta_{a b}-D_{i}\left(T_{b} z\right)^{j} D_{j}\left(T_{a} z\right)^{i}\right)+\text { h.c. }\right]+2 M \bar{M} \mathcal{D}+22 \mathcal{D}^{2} \\
& +\frac{N+5}{6}\left[\left(\mathcal{W}^{a b}+\overline{\mathcal{W}}^{a b}\right) \mathcal{D}_{a} \mathcal{D}_{b}-x\left(F_{\rho \mu}^{a}-i \tilde{F}_{\rho \mu}^{a}\right)\left(F_{a}^{\rho \nu}+i \tilde{F}_{a}^{\rho \nu}\right) \mathcal{D}_{\nu} z^{i} \mathcal{D}^{\mu} \bar{z}^{\bar{m}} K_{i \bar{m}}\right] \\
& +\frac{N+5}{3}\left[x^{2} \mathcal{W}_{a b} \overline{\mathcal{W}}^{a b}+2 \mathcal{D}^{2}-\mathcal{D} K_{i \bar{m}}\left(\mathcal{D}_{\rho} z^{i} \mathcal{D}^{\rho} \bar{z}^{\bar{m}}+2 F^{i} \bar{F}^{\bar{m}}\right)+\frac{2}{9} \mathcal{D} M \bar{M}\right] \\
& +12\left(\mathcal{W}^{a b}+\overline{\mathcal{W}}^{a b}\right) \mathcal{D}_{a} \mathcal{D}_{b}+x(\mathcal{W}+\overline{\mathcal{W}})\left[\frac{2}{3} M \bar{M}+K_{i \bar{m}}\left(\mathcal{D}_{\rho} z^{i} \mathcal{D}^{\rho} \bar{z}^{\bar{m}}-2 F^{i} \bar{F}^{\bar{m}}\right)\right] \\
& -2 x(\mathcal{W}+\overline{\mathcal{W}}) \mathcal{D}+2 \mathcal{D} K_{i \bar{m}}\left(8 \mathcal{D}_{\rho} z^{i} \mathcal{D}^{\rho} \bar{z}^{\bar{m}}+11 F^{i} \bar{F}^{\bar{m}}\right)-26 i \mathcal{D}_{\mu} z^{j} \mathcal{D}_{\nu} \bar{z}^{\bar{m}} K_{i \bar{m}} \mathcal{D}^{a} F_{a}^{\mu \nu} \\
& +14 x^{2} \mathcal{W}_{a b} \overline{\mathcal{W}}^{a b}+\left(\mathcal{D}_{\mu} z^{i} \mathcal{D}^{\mu} \bar{z}^{\bar{m}}+F^{i} \bar{F}^{\bar{m}}\right)\left[\frac{2}{x} R_{\bar{n} i \bar{m} j} \mathcal{D}_{a} D^{j}\left(T^{a} \bar{z}\right)^{\bar{n}}+\frac{4}{3} \mathcal{D} R_{i \bar{m}}\right] \\
& +\frac{D_{i}\left(T_{a} z\right)^{i}}{6 x}\left\{4 \mathcal{D}_{a}\left[\left(\mathcal{D}_{\mu} z^{j} \mathcal{D}^{\mu} \bar{z}^{\bar{m}}+F^{j} \bar{F}^{\bar{m}}\right) K_{j \bar{m}}-2 \mathcal{D}\right]+13 i F_{\mu \nu}^{a} K_{\bar{m} j} \mathcal{D}^{\mu} z^{j} \mathcal{D}^{\nu} \bar{z}^{\bar{m}}\right] \\
& +2 i F_{\mu \nu}^{a} D_{j}\left(T_{a} z\right)^{i} R_{i \bar{m} k}^{j} \mathcal{D}^{\mu} z^{k} \mathcal{D}^{\nu} \bar{z}^{\bar{m}}-\frac{e^{-K / 2}}{x} \mathcal{D}_{a}\left[\left(T^{a} z\right)^{i} R_{i}{ }^{j}{ }^{k}{ }^{k} F^{\ell} A_{j k}+\text { h.c. }\right] \\
& +\frac{f_{i} \bar{f}_{\bar{m}}}{2 x^{2}}\left(F^{i} \bar{F}^{\bar{m}}+19 \mathcal{D}_{\mu} z^{i} \mathcal{D}^{\mu} \bar{z}^{\bar{m}}\right) \mathcal{D}-\mathcal{D}\left(4+\frac{f_{k} \bar{f}^{k}}{6 x^{2}}\right)\left(\frac{f_{i}}{x} F^{i} M+\text { h.c. }\right) \\
& +\frac{\bar{f}_{\bar{m}} f_{j}}{2 x} \bar{F}^{\bar{m}} F^{j}(\mathcal{W}+\overline{\mathcal{W}})+\frac{f_{k} \bar{f}^{k} \bar{f}_{\bar{m}} f_{j}}{8 x^{4}} \mathcal{D}\left(\mathcal{D}_{\mu} \bar{z}^{\bar{m}} \mathcal{D}^{\mu} z^{j}-\bar{F}^{\bar{m}} F^{j}\right) \\
& -\frac{f_{i} \bar{f}^{\bar{m}}}{x}\left[\frac{1}{4}\left(F_{\mu \nu}^{a}+i \tilde{F}_{\mu \nu}^{a}\right)\left(F_{a}^{\mu \rho}-i \tilde{F}_{a}^{\mu \rho}\right) \mathcal{D}_{\rho} z^{i} \mathcal{D}^{\nu} \bar{z}^{\bar{m}}+\frac{11 i}{2 x} \mathcal{D}_{\mu} z^{i} \mathcal{D}^{\nu} \bar{z}^{\bar{m}} \mathcal{D}^{a} F_{a}^{\mu \nu}\right]
\end{aligned}
$$




$$
\begin{aligned}
& -\left(5+\frac{f_{k} \bar{f}^{k}}{4 x^{2}}\right)\left\{\left[\frac{i}{x}\left(F_{a}^{\nu \mu}-i \tilde{F}_{a}^{\nu \mu}\right)+\frac{g^{\nu \mu}}{x^{2}} \mathcal{D}^{a}\right] f_{j} \mathcal{D}_{\nu} z^{j} K_{i \bar{m}}\left(T^{a} z\right)^{i} \mathcal{D}_{\mu} \bar{z}^{\bar{m}}+\text { h.c. }\right\} \\
& -\frac{3 f_{k} \bar{f}^{k} f_{i} \bar{f}_{\bar{m}}}{16 x^{4}}\left[x\left(F_{\mu \nu}^{a}+i \tilde{F}_{\mu \nu}^{a}\right)\left(F_{a}^{\mu \rho}-i \tilde{F}_{a}^{\mu \rho}\right) \mathcal{D}_{\rho} z^{i} \mathcal{D}^{\nu} \bar{z}^{\bar{m}}+2 i \mathcal{D}_{\mu} z^{i} \mathcal{D}^{\nu} \bar{z}^{\bar{m}} \mathcal{D}^{a} F_{a}^{\mu \nu}\right] \\
& +\frac{\bar{f}^{i} f_{i}}{2 x^{2}} K_{i \bar{m}}\left[\mathcal{D}\left(\mathcal{D}_{\mu} z^{i} \mathcal{D}^{\mu} \bar{z}^{\bar{m}}+2 F^{i} \bar{F}^{\bar{m}}\right)+i \mathcal{D}_{\mu} z^{j} \mathcal{D}_{\nu} \bar{z}^{\bar{m}} \mathcal{D}^{a} F_{a}^{\mu \nu}\right] \\
& -\frac{\bar{f}^{i} f_{i}}{8 x^{2}}\left[8 x(\mathcal{W}+\overline{\mathcal{W}}) \mathcal{D}+\left(4 x^{2}-\bar{f}^{j} f_{j}\right) \mathcal{W}_{a b} \overline{\mathcal{W}}^{a b}+2\left(\mathcal{W}^{a b}+\overline{\mathcal{W}}^{a b}\right) \mathcal{D}_{a} \mathcal{D}_{b}\right] \\
& +\frac{f_{i} \bar{f}^{i}}{8 x^{2}}\left[x\left(F_{\rho \mu}^{a}-i \tilde{F}_{\rho \mu}^{a}\right)\left(F_{a}^{\rho \nu}+i \tilde{F}_{a}^{\rho \nu}\right) \mathcal{D}_{\nu} z^{i} \mathcal{D}^{\mu} \bar{z}^{\bar{m}} K_{i \bar{m}}-4 \mathcal{D}^{2}\right]+x^{4} \rho_{i j} \rho^{i j} \mathcal{W} \overline{\mathcal{W}} \\
& +\left\{\left(F^{j} \bar{F}^{\bar{m}}+\mathcal{D}_{\mu} z^{j} \mathcal{D}^{\mu} \bar{z}^{\bar{m}}\right)\left[4 \rho_{\bar{m} i j}\left(T^{a} z\right)^{i} \mathcal{D}_{a}-\left(\rho_{\bar{m} i j}+\frac{\bar{f}_{\bar{m}}}{x} \rho_{i j}\right) \bar{f}^{i} \mathcal{D}\right]+\text { h.c. }\right\} \\
& +e^{-K / 2}\left\{\overline{\mathcal{W}}\left[x^{2} \rho^{i j}\left(A_{j i k} F^{k}-\frac{1}{3} A_{i j} \bar{M}\right)+\frac{f_{k} \bar{f}^{i} \bar{f}^{j}}{4 x^{2}} F^{k} A_{i j}\right]+\text { h.c. }\right\} \\
& +2 x\left[4 \rho_{i j}\left(T^{a} z\right)^{i}\left(T^{b} z\right)^{j} \mathcal{W}_{a b}+i \rho_{\bar{m} i j} \mathcal{D}_{\mu} z^{j} \mathcal{D}_{\nu} \bar{z}^{\bar{m}}\left(T^{a} z\right)^{i}\left(F_{a}^{\mu \nu}-i \tilde{F}_{a}^{\mu \nu}\right)+\text { h.c. }\right] \\
& +\left\{\rho_{i j} \mathcal{D}^{\mu} z^{j}\left[\frac{2}{x} \bar{f}_{\bar{m}} \mathcal{D}_{\mu} \bar{z}^{\bar{m}}\left(T^{a} z\right)^{i} \mathcal{D}_{a}-\frac{\bar{f}^{i}}{2} \mathcal{W} f_{j} \mathcal{D}_{\mu} z^{j}\right]+\text { h.c. }\right\}+2 x^{2} \rho_{i j} \rho_{\bar{m}}^{j} \mathcal{D} \mathcal{D}_{\rho} z^{i} \mathcal{D}^{\rho} \bar{z}^{\bar{m}} \\
& -\frac{i}{2} K_{i \bar{m}}\left[\mathcal{D}^{\nu} \bar{z}^{\bar{m}}\left(T_{a} z\right)^{i}-\mathcal{D}^{\nu} z^{i}\left(T_{a} z\right)^{\bar{m}}\right]\left[\bar{f}^{i} \rho_{i j} \mathcal{D}^{\rho} z^{j}\left(F_{\rho \nu}^{a}-i \tilde{F}_{\rho \nu}^{a}\right)+\text { h.c. }\right] \\
& +\frac{\mathcal{D}_{a}}{2 x}\left[K_{k \bar{m}} \mathcal{D}^{\mu} \bar{z}^{\bar{m}}\left(T^{a} z\right)^{k}+\frac{i}{2} \bar{f}_{\bar{m}} \mathcal{D}_{\nu} \bar{z}^{\bar{m}}\left(F^{\nu \mu}-i \tilde{F}^{\nu \mu}\right)+\text { h.c. }\right]\left(\rho_{i j} \mathcal{D}_{\mu} z^{i} \bar{f}^{j}+\text { h.c. }\right) \\
& -\left[\mathcal{W}^{a b} \rho_{i j} \bar{f}^{i}\left(T_{a} z\right)^{j} \mathcal{D}_{b}+x^{2} \mathcal{D}_{\rho} z^{i} \mathcal{D}^{\rho} z^{j}\left(2 \rho_{i j} \mathcal{W}-R_{\bar{n} i \bar{m} j} \rho^{\bar{m} \bar{n}} \overline{\mathcal{W}}\right)+\text { h.c. }\right] \text {, } \\
& L_{g}=\frac{e^{-K}}{4 x^{2}}\left(\mathcal{D}_{\mu} z^{i} \mathcal{D}^{\mu} \bar{z}^{\bar{m}}+F^{i} \bar{F}^{\bar{m}}\right) A_{i j} \bar{A}_{\bar{m}}^{k} f_{k} \bar{f}^{j}+\frac{2}{9} M \bar{M}\left(\rho_{i j} \mathcal{D}_{\mu} z^{i} \mathcal{D}^{\mu} z^{j}+\text { h.c. }\right) \\
& -\frac{e^{-K / 2}}{8 x^{3}}\left\{\bar{f}^{j} A_{i j} F^{k} f_{k}\left[\bar{f}_{\bar{m}}\left(\mathcal{D}_{\mu} z^{i} \mathcal{D}^{\mu} \bar{z}^{\bar{m}}-F^{i} \bar{F}^{\bar{m}}\right)+\mathcal{D}_{\mu} z^{i} f_{\ell} \mathcal{D}^{\mu} z^{\ell}-\frac{4 x}{3} F^{i} M\right]+\text { h.c. }\right\} \\
& -\frac{e^{-K / 2}}{2}\left\{\bar{f}^{\ell} A_{k \ell}\left[\frac{\bar{f}_{\bar{m}}}{3 x^{2}} \mathcal{D}^{\mu} \bar{z}^{\bar{m}} \mathcal{D}_{\mu} z^{\ell} \bar{M}-\rho_{i j} \mathcal{D}_{\mu} z^{i}\left(F^{j} \mathcal{D}^{\mu} z^{k}-F^{k} \mathcal{D}^{\mu} z^{j}\right)\right]+\text { h.c. }\right\} \\
& +\frac{1}{2 x^{2}}\left\{\mathcal{D}_{\mu} z^{i} \bar{F}_{i}\left[\frac{x}{3} \bar{M}\left(2 \bar{f}_{\bar{m}} \mathcal{D}^{\mu} \bar{z}^{\bar{m}}-f_{j} \mathcal{D}^{\mu} z^{j}\right)+f_{j} F^{j} \bar{f}_{\bar{m}} \mathcal{D}^{\mu} \bar{z}^{\bar{m}}\right]+\text { h.c. }\right\} \\
& +\frac{1}{4 x^{2}}\left[\mathcal{D}_{\mu} z^{i} \mathcal{D}^{\mu} z^{j} f_{i} f_{j}\left(\frac{1}{9} M \bar{M}+\frac{1}{4 x^{2}} f_{k} F^{k} \bar{f}_{\bar{m}} \bar{F}^{\bar{m}}+\frac{1}{6 x} f_{k} F^{k} M\right)+\text { h.c. }\right]
\end{aligned}
$$




$$
\begin{aligned}
& -\left\{\frac{f_{i} f_{j} \bar{f}_{\bar{m}}}{24 x^{3}}\left[\left(\mathcal{D}_{\mu} z^{i} \mathcal{D}^{\mu} \bar{z}^{\bar{m}}+2 F^{i} \bar{F}^{\bar{m}}\right) F^{j} M-2 \mathcal{D}_{\mu} z^{i} \mathcal{D}^{\mu} z^{j} \bar{F}^{\bar{m}} \bar{M}\right]+\text { h.c. }\right\} \\
& -\frac{1}{4 x}\left\{\rho_{i j} \bar{f}^{\ell} \bar{F}_{\ell} \mathcal{D}_{\mu} z^{i}\left[f_{k}\left(F^{j} \mathcal{D}^{\mu} z^{k}+F^{k} \mathcal{D}^{\mu} z^{j}\right)+\bar{f}_{\bar{n}} F^{j} \mathcal{D}^{\mu} \bar{z}^{\bar{n}}+\frac{4 x}{3} \mathcal{D}^{\mu} z^{j} \bar{M}\right]+\text { h.c. }\right\} \\
& +\frac{f_{k} \bar{f}^{k}}{4 x}\left[\mathcal{W}\left(x \rho_{i j} \mathcal{D}_{\mu} z^{i} \mathcal{D}^{\mu} z^{j}-\frac{e^{-K / 2}}{2 x} f_{j} \bar{A}_{\bar{m}}^{j} \bar{F}^{\bar{m}}-\frac{\bar{f}_{\bar{m}} f_{j}}{4 x^{2}} F^{j} \bar{F}^{\bar{m}}-\frac{f_{i}}{3 x} F^{i} M\right)+\text { h.c. }\right] \\
& -\frac{1}{3} K_{i \bar{m}} K_{j \bar{n}}\left(\mathcal{D}_{\mu} z^{i} \mathcal{D}^{\mu} \bar{z}^{\bar{m}} \mathcal{D}_{\nu} z^{j} \mathcal{D}^{\nu} \bar{z}^{\bar{n}}+F^{i} \bar{F}^{\bar{m}} F^{j} \bar{F}^{\bar{n}}-F^{j} \bar{F}^{\bar{n}} \mathcal{D}_{\mu} z^{i} \mathcal{D}^{\mu} \bar{z}^{\bar{m}}\right) \\
& +\frac{1}{6} K_{i \bar{m}} K_{j \bar{n}} \mathcal{D}_{\mu} z^{i} \mathcal{D}_{\nu} \bar{z}^{\bar{m}}\left(4 \mathcal{D}^{\mu} z^{j} \mathcal{D}^{\nu} \bar{z}^{\bar{n}}+\mathcal{D}^{\nu} z^{j} \mathcal{D}^{\mu} \bar{z}^{\bar{n}}\right)-\frac{1}{27}(M \bar{M})^{2}+\frac{2}{9} \mathcal{D} M \bar{M} \\
& +\left(\frac{1}{9} M \bar{M}-\frac{1}{3} \mathcal{D}\right) K_{i \bar{m}}\left(2 F^{i} \bar{F}^{\bar{m}}-\mathcal{D}_{\mu} z^{i} \mathcal{D}^{\mu} \bar{z}^{\bar{m}}\right)+\frac{2}{3} \mathcal{D}^{2}+x^{2} \mathcal{W}_{a b} \overline{\mathcal{W}}^{a b} \\
& +\left(F_{\rho \mu}^{a}+i \tilde{F}_{\rho \mu}^{a}\right)\left(F_{a}^{\rho \nu}-i \tilde{F}_{a}^{\rho \nu}\right)\left(\frac{f_{i} \bar{f}_{\bar{m}}}{4 x}-\frac{x}{2} K_{i \bar{m}}\right) \mathcal{D}_{\nu} z^{i} \mathcal{D}^{\mu} \bar{z}^{\bar{m}}+\left(\frac{f_{i} \bar{f}^{i}}{4 x}\right)^{2} \mathcal{W} \overline{\mathcal{W}} \\
& +\frac{1}{2}\left(\mathcal{W}_{a b}+\overline{\mathcal{W}}_{a b}\right) \mathcal{D}^{a} \mathcal{D}^{b}+x^{2} \rho_{i j} \rho_{\bar{n} \bar{m}}\left(\mathcal{D}_{\mu} z^{i} \mathcal{D}^{\mu} z^{j} \mathcal{D}_{\nu} \bar{z}^{\bar{m}} \mathcal{D}^{\nu} \bar{z}^{n}+\mathcal{D}_{\mu} z^{i} \mathcal{D}^{\mu} \bar{z}^{\bar{m}} F^{j} \bar{F}^{\bar{n}}\right) \\
& +\frac{f_{i} f_{j} \bar{f}_{\bar{m}} \bar{f}_{\bar{n}}}{16 x^{4}}\left(\mathcal{D}_{\mu} z^{i} \mathcal{D}^{\mu} z^{j} \mathcal{D}_{\nu} \bar{z}^{\bar{m}} \mathcal{D}^{\nu} \bar{z}^{\bar{n}}-F^{i} F^{j} \bar{F}^{\bar{m}} \bar{F}^{\bar{n}}-2 \mathcal{D}_{\mu} z^{i} \mathcal{D}^{\mu} \bar{z}^{\bar{m}} F^{j} \bar{F}^{\bar{n}}\right) \\
& +\frac{f_{j} \bar{f}_{\bar{n}}}{12 x^{2}} F^{j} \bar{F}^{\bar{n}}\left[K_{i \bar{m}}\left(\mathcal{D}_{\mu} z^{i} \mathcal{D}^{\mu} \bar{z}^{\bar{m}}-2 F^{i} \bar{F}^{\bar{m}}\right)+2 M \bar{M}-2 \mathcal{D}\right] \\
& +\frac{f_{j} \bar{f}_{\bar{n}}}{6 x^{2}} \mathcal{D}_{\nu} z^{j} \mathcal{D}^{\nu} \bar{z}^{\bar{n}}\left[K_{i \bar{m}}\left(2 \mathcal{D}_{\mu} z^{i} \mathcal{D}^{\mu} \bar{z}^{\bar{m}}-F^{i} \bar{F}^{\bar{m}}\right)+\frac{1}{2} M \bar{M}-\mathcal{D}\right] \\
& -\frac{f_{j} \bar{f}_{\bar{m}}}{2 x^{2}} K_{j \bar{n}}\left(\mathcal{D}_{\mu} z^{i} \mathcal{D}^{\mu} z^{j} \mathcal{D}_{\nu} \bar{z}^{\bar{m}} \mathcal{D}^{\nu} \bar{z}^{\bar{n}}+\mathcal{D}_{\mu} z^{i} \mathcal{D}^{\mu} \bar{z}^{\bar{n}} \mathcal{D}_{\nu} \bar{z}^{\bar{m}} \mathcal{D}^{\nu} z^{j}\right)
\end{aligned}
$$

where indices are raised and lowered with the Kähler metric $\left(\bar{F}_{i}=K_{i \bar{m}} \bar{F}^{\bar{m}}\right.$, etc. $)$, and

$$
\mathcal{W}_{b}^{a}=\frac{1}{4}\left(F_{\mu \nu}^{a} F_{b}^{\mu \nu}-i F_{\mu \nu}^{a} \tilde{F}_{b}^{\mu \nu}\right)-\frac{1}{2 x^{2}} \mathcal{D}^{a} \mathcal{D}_{b}, \quad \mathcal{W}=\mathcal{W}_{a}^{a}
$$

is the bosonic part of the $F$-component of the composite chiral supermultiplet constructed from the Yang-Mills chiral superfield $W^{a}(\theta)=\lambda_{L}^{a}+O(\theta), F^{i}=$ $-e^{-K / 2} \bar{A}^{i}$ is the bosonic part of the $F$-component of the chiral supermultiplet $Z^{i}, M=-3 e^{-K / 2} A$ is an auxiliary field in the gravity supermultiplet [ $[$ ]. The results of [1, 2] were calculated using the classical Lagrangian (1) that is obtained after elimination of the auxiliary fields, and are expressed in 
those papers as functions of the boson fields and their covariant derivatives. It is easy to show that calculating the one loop corrections before or after elimination of the auxiliary fields in terms of their classical solutions gives the same result to the loop order considered. Here we use the auxiliary fields to present the results in a form that lends itself more easily to an interpretation in terms of superfield operators.

The real function $K_{R}(Z, \bar{Z})$,

$$
K_{R}=K+\frac{\ln \Lambda^{2}}{32 \pi^{2}}\left[e^{-K}\left(A_{i j} \bar{A}^{i j}-2 A_{i} \bar{A}^{i}-4 A \bar{A}\right)-4 \mathcal{K}_{a}^{a}-\left(12+4 x^{2} \rho_{i} \rho^{i}\right) \mathcal{D}\right]
$$

contains logarithmically divergent contributions to the the renormalized Kähler potential. It was shown in [12] that the quadratically divergent term, after Pauli-Villars regularization and an appropriate Weyl transformation, can be absorbed into an additional renormalization of the Kähler potential. In writing $L_{1,2, g}$ we have dropped total derivatives, as well as terms that vanish when the tree-level equations of motion are imposed, including a redefinition [1] of the space-time metric, so as to cast the Einstein term in canonical form. In $L_{2, g}$ we have also introduced scalar field reparameterization invariant covariant derivatives $\left(\rho_{i j}, \rho_{\bar{m} i j}\right)$ of the variable $\rho$, defined as the squared gauge coupling $\rho=x^{-1}=g^{2}$.

In effective supergravity from superstring theory, the classical Kähler potential $K(z, \bar{z})$, superpotential $W(z)$ and Yang-Mills normalization function $f_{a b}(z)$ take the forms

$$
\begin{aligned}
K(z, \bar{z}) & =-\ln (s+\bar{s})+G(y, \bar{y}), \quad W(z)=W(y), \\
f_{a b}(z) & =\delta_{a b} k_{a} s, \quad s=z^{0}, y^{i}=z^{i \geq 1} .
\end{aligned}
$$

In this case $1-(4 x)^{-2} \bar{f}^{i} f_{i}=A+(2 x)^{-1} \bar{f}^{i} A_{i}=0$, and $\rho_{i}=D_{i} \rho=-\left(2 x^{2}\right)^{-1} f_{i}$ is covariantly constant: $\rho_{i j}=\rho_{\bar{m} i j}=\cdots=0$. Then $L_{2}$ and $L_{g}$ reduce to:

$$
\begin{aligned}
L_{2}= & \left(\mathcal{W}^{a b}+\overline{\mathcal{W}}^{a b}\right)\left[3 C_{G} \delta_{a b}-D_{i}\left(T_{b} z\right)^{j} D_{j}\left(T_{a} z\right)^{i}\right]-24 i \mathcal{D}_{\mu} z^{i} \mathcal{D}_{\nu} \bar{z}^{\bar{m}} K_{i \bar{m}} \mathcal{D}^{a} F_{a}^{\mu \nu} \\
& +\frac{N+5}{12}\left[(s+\bar{s})^{2} \mathcal{W}_{a b} \overline{\mathcal{W}}^{a b}+2\left(\mathcal{W}^{a b}+\overline{\mathcal{W}}^{a b}\right) \mathcal{D}_{a} \mathcal{D}_{b}+8 \mathcal{D}^{2}\right]+\frac{7}{2}(s+\bar{s})^{2} \mathcal{W}_{a b} \overline{\mathcal{W}}^{a b}
\end{aligned}
$$




$$
\begin{aligned}
& -\frac{N+5}{3} \mathcal{D}\left[K_{i \bar{m}}\left(2 F^{i} \bar{F}^{\bar{m}}+\mathcal{D}_{\mu} z^{i} \mathcal{D}^{\mu} \bar{z}^{\bar{m}}\right)-\frac{2}{9} M \bar{M}\right]+11\left(\mathcal{W}^{a b}+\overline{\mathcal{W}}^{a b}\right) \mathcal{D}_{a} \mathcal{D}_{b} \\
& +\frac{(s+\bar{s})}{2}(\mathcal{W}+\overline{\mathcal{W}})\left[\frac{2}{3} M \bar{M}+K_{i \bar{m}}\left(\mathcal{D}_{\rho} z^{i} \mathcal{D}^{\rho} \bar{z}^{\bar{m}}-2 F^{i} \bar{F}^{\bar{m}}\right)+2 \mathcal{D}\right]+20 \mathcal{D}^{2} \\
& -\frac{N+2}{12}(s+\bar{s})\left(F_{\rho \mu}^{a}-i \tilde{F}_{\rho \mu}^{a}\right)\left(F_{a}^{\rho \nu}+i \tilde{F}_{a}^{\rho \nu}\right) \mathcal{D}_{\nu} z^{i} \mathcal{D}^{\mu} \bar{z}^{\bar{m}} K_{i \bar{m}}+\frac{154}{9} M \bar{M} \mathcal{D} \\
& +2 K_{i \bar{m}}\left(13 F^{i} \bar{F}^{\bar{m}}+9 \mathcal{D}_{\mu} z^{i} \mathcal{D}^{\mu} \bar{z}^{\bar{m}}\right) \mathcal{D}-\frac{2 e^{-K / 2}}{(s+\bar{s})} \mathcal{D}_{a}\left[\left(T^{a} z\right)^{i} R_{i}{ }^{j}{ }^{k} F^{\ell} A_{j k}+\text { h.c. }\right] \\
& -\left[\frac{4}{3} \mathcal{D} R_{i \bar{m}}+\frac{\mathcal{D}_{a}}{(s+\bar{s})}\left(R_{\bar{n} i \bar{m} j} D^{j}\left(T^{a} \bar{z}\right)^{\bar{n}}-\frac{4}{3} K_{i \bar{m}} D_{j}\left(T^{a} z\right)^{j}\right)\right]\left(F^{i} \bar{F}^{\bar{m}}+\mathcal{D}_{\mu} z^{i} \mathcal{D}^{\mu} \bar{z}^{\bar{m}}\right) \\
& +\frac{D_{j}\left(T^{a} z\right)^{j}}{3}\left[13 i K_{\bar{m} i} F_{\mu \nu}^{a} \mathcal{D}^{\mu} z^{i} \mathcal{D}^{\nu} \bar{z}^{\bar{m}}-\frac{8 \mathcal{D}_{a} \mathcal{D}}{(s+\bar{s})}\right]+2 i F_{\mu \nu}^{a} D_{j}\left(T_{a} z\right)^{i} R_{i \bar{m} k}^{j} \mathcal{D}^{\mu} z^{k} \mathcal{D}^{\nu} \bar{z}^{\bar{m}} \\
& -\frac{12}{s+\bar{s}}\left\{\left[i \partial_{\nu} s\left(F_{a}^{\nu \mu}-i \tilde{F}_{a}^{\nu \mu}\right)+\frac{2 \partial^{\mu} s}{s+\bar{s}} \mathcal{D}_{a}\right] \mathcal{D}_{\mu} \bar{z}^{\bar{m}} K_{i \bar{m}}\left(T^{a} z\right)^{i}+\text { h.c. }\right\} \\
& -2 \frac{\partial_{\rho} s \partial^{\nu} \bar{s}}{(s+\bar{s})}\left(F_{\mu \nu}^{a}+i \tilde{F}_{\mu \nu}^{a}\right)\left(F_{a}^{\mu \rho}-i \tilde{F}_{a}^{\mu \rho}\right)+40 \frac{\partial_{\mu} s \partial^{\mu} \bar{s}}{(s+\bar{s})^{2}} \mathcal{D}+28 i \frac{\partial_{\mu} s \partial_{\nu} \bar{s}}{(s+\bar{s})^{2}} \mathcal{D}^{a} F_{a}^{\mu \nu}, \\
& L_{g}=\frac{(s+\bar{s})^{2}}{4}\left(\mathcal{W} \overline{\mathcal{W}}+\mathcal{W}_{a b} \overline{\mathcal{W}}^{a b}\right)+\frac{1}{2}\left(\mathcal{W}_{a b}+\overline{\mathcal{W}}_{a b}\right) \mathcal{D}^{a} \mathcal{D}^{b}+\frac{2}{3} \mathcal{D}^{2} \\
& +\frac{1}{6} K_{i \bar{m}} K_{j \bar{n}}\left(4 \mathcal{D}_{\mu} z^{i} \mathcal{D}^{\mu} z^{j} \mathcal{D}_{\nu} \bar{z}^{\bar{m}} \mathcal{D}^{\nu} \bar{z}^{\bar{n}}+\mathcal{D}_{\mu} z^{i} \mathcal{D}^{\mu} \bar{z}^{\bar{n}} \mathcal{D}_{\nu} \bar{z}^{\bar{m}} \mathcal{D}^{\nu} z^{j}\right) \\
& -\frac{1}{3} K_{i \bar{m}} K_{j \bar{n}}\left(\mathcal{D}_{\mu} z^{i} \mathcal{D}^{\mu} \bar{z}^{\bar{m}} \mathcal{D}_{\nu} z^{j} \mathcal{D}^{\nu} \bar{z}^{\bar{n}}+F^{i} \bar{F}^{\bar{m}} F^{j} \bar{F}^{\bar{n}}-F^{j} \bar{F}^{\bar{n}} \mathcal{D}_{\mu} z^{i} \mathcal{D}^{\mu} \bar{z}^{\bar{m}}\right) \\
& +\left(\frac{2}{27} M \bar{M}-\frac{1}{3} \mathcal{D}\right) K_{i \bar{m}}\left(2 F^{i} \bar{F}^{\bar{m}}-\mathcal{D}_{\mu} z^{i} \mathcal{D}^{\mu} \bar{z}^{\bar{m}}\right)-\frac{2}{27} \mathcal{D} M \bar{M} \\
& -\frac{1}{81}(M \bar{M})^{2}-(s+\bar{s})(\mathcal{W}+\overline{\mathcal{W}})\left(\frac{1}{2} F^{i} \bar{F}^{\bar{m}} K_{i \bar{m}}-\frac{1}{9} M \bar{M}\right) \\
& +\frac{2 \partial_{\nu} s \partial^{\nu} \bar{s}}{3(\bar{s}+\bar{s})^{2}}\left[K_{i \bar{m}}\left(2 \mathcal{D}_{\mu} z^{i} \mathcal{D}^{\mu} \bar{z}^{\bar{m}}-F^{i} \bar{F}^{\bar{m}}\right)+\frac{1}{3} M \bar{M}-\mathcal{D}\right] \\
& +\frac{1}{(s+\bar{s})^{4}} \partial_{\mu} s \partial^{\mu} s \partial_{\nu} \bar{s} \partial^{\nu} \bar{s}-\frac{2 \partial_{\mu} s \partial_{\nu} \bar{s}}{(s+\bar{s})^{2}} K_{j \bar{n}}\left(\mathcal{D}^{\mu} z^{j} \mathcal{D}^{\nu} \bar{z}^{\bar{n}}+\mathcal{D}^{\mu} \bar{z}^{\bar{n}} \mathcal{D}^{\nu} z^{j}\right) \\
& +\left(F_{\rho \mu}^{a}+i \tilde{F}_{\rho \mu}^{a}\right)\left(F_{a}^{\rho \nu}-i \tilde{F}_{a}^{\rho \nu}\right)\left(\frac{\partial_{\nu} s \partial^{\mu} \bar{s}}{2(s+\bar{s})}-\frac{s+\bar{s}}{4} K_{i \bar{m}} \mathcal{D}_{\nu} z^{i} \mathcal{D}^{\mu} \bar{z}^{\bar{m}}\right),
\end{aligned}
$$


with now:

$$
\begin{aligned}
K_{R} & =K+\frac{\ln \Lambda^{2}}{32 \pi^{2}}\left\{e^{-K}\left[A_{i j} \bar{A}^{i j}-2 A_{i} \bar{A}^{i}+\left(N_{G}-4\right) A \bar{A}\right]-4 \mathcal{K}_{a}^{a}-16 \mathcal{D}\right\} \\
M & =-\frac{3}{s+\bar{s}} \bar{F}^{\bar{s}}, \quad M \bar{M}=9 F^{s} \bar{F}_{s}
\end{aligned}
$$

where the second line follows from the tree level equations of motion.

In addition, in the untwisted sector of orbifold compactifications, the Riemann tensor is covariantly constant and its elements are related to elements of the Kähler metric. Moreover in many models there are global symmetries that impose $R^{i \bar{m} j \bar{n}} W_{i j}=R^{i \bar{m} j \bar{n}} W_{i j k}=0$. In this case $L_{1}$ can be expressed entirely in terms of $F^{i}, \mathcal{D}_{\mu} z^{i}, M$, their complex conjugates, and the Kähler metric; an explicit example is given in [1].

Some comments on the implications and applications of our results are in order. It has already been shown, using the gauge fixing and expansion procedures defined here, that the one-loop quadratic divergences [12], as well as the logarithmic divergences [15] in the flat space limit and in the absence of a dilaton, can be regulated à la Pauli-Villars. Regularization of the full supergravity divergences without a dilaton are under study [16]. An objective of this study is to determine the extent to which a regularization procedure can be achieved that preserves nonlinear symmetries of the classical effective Lagrangian. To obtain the full one-loop Lagrangian, including finite contributions, requires a resummation of the derivative expansion. A procedure for resummation will be described elsewhere [16].

We have presented our results for one-loop corrections to the general

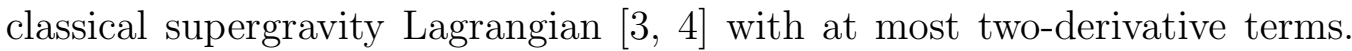
Our results for this general case can be summarized as follows. We define an operator of dimension $d$ as a Kähler invariant operator whose term of lowest dimension is $d$, where scalar and Yang-Mills fields are assigned the canonical dimension of unity. Then, among the ultra-violet divergent terms generated at one loop, all operators of dimension 6 or less (as well as many operators of dimension 8) that involve neither the Kähler curvature 
nor derivatives of the gauge kinetic function can be absorbed by field redefinitions, interpreted as renormalizations of the Kähler potential, or take the form $F_{a b}(z, \bar{z})\left(W^{a} W^{b}\right)_{F}+$ h.c., where $W^{a}$ is a chiral Yang-Mills supermultiplet, the subscript denotes the F-component, and the matrix-valued function $F_{a b}(z, \bar{z})$ is not in general holomorphic. The remaining terms of dimension 8 and higher must be interpreted as arising from higher order spinorial derivatives of superfield operators.

As seen above, the result simplifies considerably for the classical effective Lagrangian derived from string theory, due to the the absence of a potential for the dilaton and the special form of its Kähler potential. These features are modified when the effective Lagrangian includes a nonperturbatively induced [17] superpotential for the dilaton and/or the Green-Schwarz counterterm [18 that is necessary to restore modular invariance. The latter term destroys the no-scale nature of Lagrangians from torus compactification and the untwisted sector of orbifold compactification, and generally destabilizes the effective scalar potential. However this term is of one-loop order and therefore should be considered together with the full one-loop corrections. An interesting question, that will be addressed elsewhere, is whether the one-loop corrections presented here can restabilize the potential.

An important unresolved issue in the construction of effective supergravity Lagrangians for gaugino condensation is the correct form of the kinetic term for the composite chiral multiplet that represents the lightest bound state of the confined Yang-Mills sector. It has recently been shown [19, in the context of both the linear and chiral multiplet formulations for the dilaton, that such terms can be generated by operators of higher dimension. The effective Lagrangian (17) determines the leading one-loop contribution to the relevant operators; similar operators occur in string theory 20. Thus the determination of loop corrections can provide a guide to the construction of the effective theory, which in turn could shed light on gaugino condensation as a mechanism for supersymmetry breaking. 
Acknowledgements. This work was supported in part by the Director, Office of Energy Research, Office of High Energy and Nuclear Physics, Division of High Energy Physics of the U.S. Department of Energy under Contract DE-AC03-76SF00098 and in part by the National Science Foundation under grant PHY-95-14797 and PHY-93-09888.

\section{References}

[1] M.K. Gaillard and V. Jain, Phys. Rev. D49: 1951 (1994); misprints and errors in this paper are corrected in [2].

[2] M.K. Gaillard, V. Jain and K. Saririan, LBL-34948, UCB-PTH-93/37, ITP-SB-95-38 (1995) hep-th/9606052.

[3] E. Cremmer, S. Ferrara, L. Girardello, and A. Van Proeyen, Nucl. Phys. B212: 413 (1983).

[4] P. Binétruy, G. Girardi, R. Grimm and M. Muller, Phys. Lett. 189B: 83 (1987); P. Binétruy, G. Girardi and R. Grimm, Annecy preprint LAPPTH-275-90, (1990).

[5] R. Barbieri, S. Ferrara, L. Maiani, F. Palumbo and C.A. Savoy, Phys. Lett. 115B: 212 (1982).

[6] I. Antoniadis, E. Gava, K.S. Narain and T.R. Taylor, Nucl. Phys. B407: 706 (1993).

[7] G.K. 't Hooft and M. Veltman, Ann. Inst. Henri Poincaré 20: 69 (1974).

[8] M.K. Gaillard and V. Jain, Phys. Rev. D46: 1786 (1992).

[9] R. Barbieri and C. Cecotti, Z. Phys. C17: 183 (1983); P. Binétruy and M.K. Gaillard, Nucl. Phys. B254: 388 (1985).

[10] J. W. Burton, M.K. Gaillard and V. Jain, Phys. Rev. D41: 3118 (1990). 
[11] C. Chiou-Lahanas, A. Kapella-Economu, A.B. Lahanas and X.N. Maintas, Phys. Rev. D42: 469 (1990) and Phys. Rev. D45: 534 (1992).

[12] M.K. Gaillard, Phys.Lett. B342: 125 (1995).

[13] M.A. Shifman, Nucl. Phys. B352: 87 (1991); M.A. Shifman, and A.I. Vainshtein Nucl. Phys. B365: 312 (1991); A.A. Iogansen, Sov. J. Nucl. Phys. 54 (1991).

[14] E.S. Fradkin and A.A. Tseytlin, Ann. Phys. 162: 31 (1985). This paper showed equivalence up to finite topological anomalies; subsequently full equivalence has been shown; S.J. Rey, private communication.

[15] M.K. Gaillard, Phys. Lett B347: 284 (1995).

[16] M.K. Gaillard and S.-J. Rey, in progress.

[17] H.P. Nilles, Phys. Lett. 115B: 455 (1982).

[18] G.L. Cardoso and B.A. Ovrut, Nucl. Phys. B369: 351 (1992); J.-P. Derendinger, S. Ferrara, C. Kounnas and F. Zwirner, Phys. Lett. B271: 307 (1991); P. Binétruy, G. Girardi, R. Grimm and M. Müller, Phys. Lett. B265 111 (1991); P. Adamietz, P. Binétruy, G. Girardi and R. Grimm, Nucl. Phys. B401: 257 (1993); P. Mayr and S. Stieberger, Nucl. Phys. B412: 502 (1994); M.K. Gaillard and T.R. Taylor, Nucl. Phys. B381: 577 (1992); V. Kaplunovsky and J. Louis, Nucl. Phys. B422: 57 (1994).

[19] P. Binétruy, M.K. Gaillard and T.R. Taylor, Nucl. Phys. B455: 97 (1995); P. Binétruy and M.K. Gaillard, Phys. Lett. B365: 87 (1996).

[20] I. Antoniadis, E. Gava, K.S. Narain and T.R. Taylor, Nucl. Phys. 428: 282 (1994). 